\title{
Inatividade física em idosos não institucionalizados: estudo de base populacional
}

\author{
Physical inactivity among non-institutionalized elderly individuals: \\ a population-based study
}

Bruno Morbeck de Queiroz ${ }^{1}$
Raildo da Silva Coqueiro $^{2}$
João de Souza Leal Neto ${ }^{1}$
Adriano Ferreti Borgatto $^{3}$
Aline Rodrigues Barbosa $^{4}$
Marcos Henrique Fernandes $^{2}$

${ }^{1}$ Programa de Pós-

Graduação em Educação

Física, Centro de Desportos,

Universidade Federal de

Santa Catarina. Campus

Universitário Reitor João

David Ferreira Lima,

Trindade. 88.040-900

Florianópolis SC Brasil.

morbeck.bruno@gmail.com

${ }^{2}$ Núcleo de Estudos

em Epidemiologia

do Envelhecimento,

Departamento de Saúde,

Universidade Estadual

do Sudoeste da Bahia.

${ }^{3}$ Departamento de

Informática e Estatística,

Centro Tecnológico,

Universidade Federal de

Santa Catarina.

${ }^{4}$ Centro de Desportos,

Universidade Federal de

Santa Catarina.

\begin{abstract}
The scope of this study is to assess the prevalence of physical inactivity among the elderly in Northeast Brazil and analyze the associated lifestyle and socio-demographic factors and health conditions. The work presented here is a descriptive and association-based study using secondary data derived from a population-based epidemiological study that included 316 elderly participants. The physical activity level of each participant was assessed using the International Physical Activity Questionnaire. The explanatory variables examined in this study were gender, age group, family set-up, ability to read and write, paid or voluntary employment, smoking, symptoms of depression, high blood pressure, fasting hyperglycemia and functional capacity. The prevalence of physical inactivity was 46.5 . The results of the multivariate analysis indicated that physical inactivity was positively associated with the age group $\geq 80$ years ( $P R$ $=2.37)$, with the lack of an occupation $(P R=4.86)$ and with dependence on instrumental activities of daily life $(P R=1.47)$ and basic activities of daily life $(P R=1.60)$. Physical inactivity was highly prevalent in the surveyed population, making it essential to discuss programs that encourage and enable increased physical activity to combat the risk factors of a sedentary lifestyle.
\end{abstract}

Key words Motor activity, Aging, Epidemiology
Resumo Oobjetivo deste estudo é descrever a prevalência de inatividade física e analisar os fatores sociodemográficos, de estilo de vida e condições de saúde associados em idosos do Nordeste brasileiro. Estudo descritivo e de associação, baseado em dados secundários originados de uma pesquisa epidemiológica de base populacional envolvendo 316 idosos. O nível de atividade física foi avaliado por meio do International Physical Activity Questionnaire. As variáveis explanatórias foram: sexo, grupo etário, arranjo familiar, sabe ler e escrever, trabalho remunerado ou voluntário, tabagismo, sintomatologia depressiva, pressão arterial elevada, hiperglicemia em jejum e capacidade funcional. A prevalência da inatividade fisica foi de 46,5. Os resultados da análise múltipla indicaram que a inatividade física foi positivamente associada ao grupo etário $\geq 80$ anos $(R P=2,37)$, a falta de ocupação $(R P=4,86)$ $e$ a dependência nas atividades instrumentais de vida diária $(R P=1,47)$ e nas atividades básicas de vida diária $(R P=1,60)$. A inatividade física foi altamente prevalente na população estudada, tornando-se imprescindivel a discussão de programas que incentivem e possibilite maior adesão à prática da atividade fisica, tendo em vista o combate a inatividade física e os fatores de risco decorrentes deste comportamento.

Palavras-chave Atividade motora, Envelhecimento, Epidemiologia 


\section{Introdução}

No Brasil, o envelhecimento populacional vem ocorrendo de forma acelerada nas últimas décadas. As projeções apontam que até 2025 o país terá a sexta população de idosos do mundo, com cerca de 32 milhões de pessoas ${ }^{1}$. Esta perspectiva repercute em maior demanda por serviços de saúde, uma vez que a prevalência de doenças crônicas e limitações funcionais são maiores nesta parcela da população. Estes fatores configuram um novo panorama de atenção à saúde, marcado por problemas de saúde de longa duração e utilização de procedimentos terapêuticos de alto custo ${ }^{2}$.

A atenção integral à saúde do idoso deve incentivar o envelhecimento ativo, no qual as oportunidades à saúde são favorecidas, visando a participação e segurança de modo a realçar a qualidade de vida no envelhecimento ${ }^{3}$. O envelhecimento saudável pressupõe a interação entre aspectos dos determinantes sociais, os aspectos físicos, mentais, independência nas atividades diárias, integração social, suporte familiar e independência econômica ${ }^{4,5}$. Dentre as possibilidades para a promoção da saúde do idoso é indiscutível o papel da atividade física. Estudos de revisão e de meta-análise, assim como documentos de organizações científicas, evidenciam os efeitos preventivos e/ou terapêuticos da atividade física em relação às doenças metabólicas e cardiovasculares, câncer, depressão, lesões/fraturas por quedas e/ou osteoporose, limitação funcional e incapacidades, função cognitiva, além de melhorar a qualidade do sono ${ }^{6-8}$.

As informações referentes aos benefícios da atividade física em indivíduos idosos são consistentes. Contudo, dados de vigilância e monitoramento em atividade física têm apontando para altos índices de inatividade física em idosos em diferentes regiões do mundo ${ }^{9,10}$. O Brasil, de igual modo, tem se caracterizado por apresentar dados preocupantes quanto à prevalência de inatividade física em diferentes faixas etárias, inclusive na população idosa ${ }^{11-13}$. Poucos estudos de monitoramento foram realizados com populações de baixa renda ${ }^{14,15}$, dificultando a implementação de políticas e ações que atendam de forma eficiente às demandas dessas regiões.

Assim, considerando a importância do monitoramento dos níveis de atividade física em indivíduos idosos, o presente trabalho teve como objetivo avaliar a inatividade física e sua associação com fatores sociodemográficos, estilo de vida e condições de saúde, em idosos do nordeste brasileiro.

\section{Método}

Este é um estudo descritivo e de associação, baseado em dados secundários derivados de uma pesquisa epidemiológica transversal de base populacional e domiciliar, denominada Estado nutricional, comportamentos de risco e condições de saúde dos idosos de Lafaiete Coutinho-BA. Detalhes sobre o local e população do estudo, bem como sobre a coleta de dados, foram publicados previamente ${ }^{16,17}$. Recentemente, um censo foi conduzido em Lafaiete Coutinho (janeiro de 2011) para a identificação de idosos ( $\geq 60$ anos). A localização das residências foi realizada usando informações da Estratégia Saúde da Família, que cobre 100\% do município. Todos os idosos residentes na zona urbana $(\mathrm{N}=$ 355) foram contatados. Dos 355 indivíduos que compunham a população do estudo, 316 (89\%) participaram da pesquisa: foram registradas 17 $(4,8 \%)$ recusas e $22(6,2 \%)$ indivíduos não foram localizados após três visitas domiciliares em dias alternados e foram considerados como perdas. $\mathrm{O}$ estudo foi realizado de acordo com a Declaração de Helsinki da Associação Médica Mundial e foi aprovado pelo Comitê de Ética em Pesquisa da Universidade Estadual do Sudoeste da Bahia.

\section{Nível de atividade física (variável dependente)}

O instrumento utilizado para avaliar o nível de atividade física habitual foi o International Physical Activity Questionnaire - IPAQ, versão longa ${ }^{18}$. Foi construído um escore de atividade física em minutos/semana, sendo somados os minutos despendidos em caminhada e atividades moderadas, com os minutos despendidos em atividades de intensidade vigorosa, multiplicados por dois ${ }^{11}$. Tal estratégia visa considerar a intensidade de cada atividade e está de acordo com as recomendações atuais quanto à prática de atividade física. A classificação quanto ao nível de atividade física obedeceu ao seguinte critério ${ }^{11}$ : escore $<150$ minutos $=$ inativo e escore $\geq 150$ minutos $=$ ativo .

\section{Variáveis explanatórias}

Sociodemográficas: sexo, grupo etário (60-69, 70-79 e $\geq 80$ anos), arranjo familiar (vive acompanhado e vive sozinho) e sabe ler e escrever um recado (sim e não).

Estilo de vida: trabalho remunerado ou voluntário (sim e não), tabagismo (fumante, ex-fumante e nunca fumou) e consumo de bebidas alcóolicas ( $<1 \mathrm{dia} /$ semana e $\geq 1$ dia/semana). 


\section{Condições de saúde}

A Escala de Depressão Geriátrica (forma abreviada de 15 itens) foi usada para verificar os sintomas depressivos: $<6$ pontos $=$ negativo (ausência de sintomas depressivos) e $\geq 6$ pontos $=$ positivo (presença de sintomas depressivos) ${ }^{19}$. A pressão arterial foi aferida utilizando monitor de pressão arterial digital automático (Omron Healthcare HEM-742INT, China), de acordo com procedimentos padronizados ${ }^{20}$. A pressão arterial elevada ( sistólica $\geq 140 \mathrm{mmHg}$ e/ou diastólica $\geq$ $90 \mathrm{mmHg}$ e/ou uso de medicamento para controle da pressão arterial) foi definida de acordo com diretrizes vigentes no Brasil ${ }^{20}$. A glicemia plasmática em jejum de 12 horas foi dosada por meio do sistema Accutrend ${ }^{\varpi}$ Plus (Roche Diagnostics, Alemanha), analisador previamente validado ${ }^{21}$. As amostras de sangue capilar foram coletadas por meio de punção trans cutânea, no lado medial, da ponta do dedo médio, usando-se lanceta hipodérmica descartável. Previamente à punção, aplicava-se álcool a $70 \%$ com o intuito de promover a antissepsia do local. A glicemia de jejum foi considerada elevada $(\geq 126 \mathrm{mg} / \mathrm{dl}$ e/ou uso de medicamento oral para controle da glicemia e/ou uso de insulina), de acordo com sa recomendações da sociedade Brasileira de Diabetes ${ }^{22}$. A capacidade funcional foi avaliada por meio das informações das atividades básicas da vida diária $(\mathrm{ABVD})^{23}$ e das atividades instrumentais da vida diária (AIVD) ${ }^{24}$, conforme descrito previamente ${ }^{25}$. Foi construída uma escala de incapacidade funcional hierárquica distinguindo três categorias ${ }^{26}$ : independente, dependente nas AIVD, dependente nas ABVD e AIVD. Os idosos que relataram dependência nas $\mathrm{ABVD}$, mas não nas AIVD, foram classificados na última categoria, referente à dependência em ambas as dimensões.

\section{Procedimento estatístico}

Com o objetivo de comparar prevalências, a associação entre nível de atividade física e as variáveis explanatórias foram verificadas mediante a obtenção de estimativas brutas e ajustadas das razões de prevalências, por ponto e por intervalo de confiança de 95\% (IC95\%), por meio do modelo de regressão de Poisson. Nas análises brutas, a prevalência de sedentarismo foi calculada para cada categoria das variáveis explanatórias, e o nível de significância foi testado por meio do teste de Wald para heterogeneidade. $\mathrm{Na}$ análise ajustada, foram incluídas as variáveis que apresentaram significância estatística de pelo menos $10 \%$ ( $\mathrm{p} \leq$ $0,10)$ nas análises brutas, seguindo a ordem de um modelo hierárquico para determinação dos desfechos ${ }^{27}$, como apresentado na Figura 1. De acordo com o modelo estabelecido, as variáveis dos níveis mais superiores (distais) interagem entre si e determinam as variáveis dos níveis mais inferiores (proximais). O efeito de cada variável explanatória sobre o desfecho foi controlado para as variáveis do mesmo nível e de níveis superiores no modelo, sendo que o critério estatístico de permanência no modelo foi de $10 \%$ ( $\mathrm{p} \leq 0,10)$. Foi considerada associação significativa, variáveis que apresentaram o nível de significância adotado no estudo de no máximo $5 \%$.

Os dados foram tabulados e analisados no IBM SPSS Statistics for Windows (IBM SPSS. 21.0, 2012, Armonk, NY: IBM Corp.).

\section{Resultados}

Participaram do estudo 173 mulheres (54,7\%) e 143 homens (45,3\%), com idade entre 60 e 105 anos (74,2 $\pm 9,8$ anos). As demais características da população estudada são descritas na Tabela $1 . \mathrm{Ob}$ serva-se que a maioria dos indivíduos relatou viver acompanhado, não saber ler e escrever um recado, não ter trabalho remunerado ou voluntário, não fazer uso de bebidas alcoólicas e não apresentar sintomas depressivos. A frequência de pressão arterial elevada foi alta e de hiperglicemia baixa.

O nível de atividade física foi avaliado em 310 indivíduos $(98,1 \%)$ e a prevalência de inatividade física foi de 46,5\%. Os dados da Tabela 2 mostram a prevalência de inatividade física, de acordo com as variáveis explanatórias. A inatividade física foi significativamente superior no grupo etário $\geq 80$ anos, nos indivíduos não alfabetizados, sem trabalho remunerado ou voluntário, com sintomas depressivos e que apresentaram dependência funcional.

Os resultados da análise bruta mostraram que as variáveis explanatórias (grupo etário, saber ler e escrever, trabalho remunerado ou voluntário, consumo de bebidas alcoólicas, sintomatologia depressiva, pressão arterial elevada e capacidade funcional) alcançaram significância estatística suficiente $(\mathrm{p} \leq 0,10)$ para serem incluídas no modelo múltiplo.

A Tabela 3 apresenta os resultados da análise ajustada para a inatividade física, em relação às variáveis explanatórias do estudo. Após os ajustes intra e interníveis, de acordo com o modelo hierárquico, as variáveis saber ler e escrever consumo de bebidas alcoólicas e pressão arterial elevada não permaneceram no modelo final, por não 


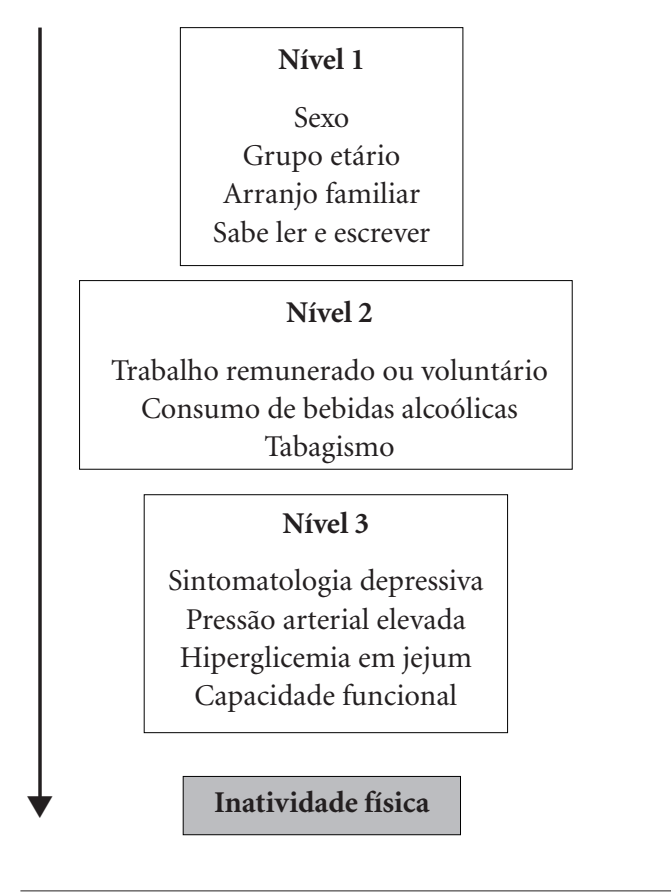

Figura 1. Modelo conceitual de determinação do desfecho utilizado na análise ajustada. Lafaiete Coutinho, Brasil, 2011.

atenderem ao critério de significância $(\mathrm{p} \leq 0,10)$. A inatividade física foi positivamente associada ao grupo etário $\geq 80$ anos, a não ter trabalho remunerado ou voluntário e à dependência funcional. Embora tenha permanecido no modelo final para fins de ajuste, a sintomatologia depressiva não foi preditora de inatividade física.

\section{Discussão}

Este estudo avaliou a prevalência de inatividade física e sua associação com fatores sociodemográficos, estilo de vida e condições de saúde, em idosos não institucionalizados. Os resultados mostraram que a inatividade física, prevalente em aproximadamente metade da população estudada, foi diretamente associada à idade, a ausência de ocupação e a dependência funcional.

Comparando a prevalência do desfecho no presente estudo com a de uma pesquisa de grande abrangência que utilizou o mesmo critério de avaliação dos níveis de atividade física ${ }^{15}$, observa-se prevalência da inatividade física similar à verificada na população idosa de cidades do Rio
Tabela 1. Características da população estudada. Lafaiete Coutinho, Brasil, 2011.

\begin{tabular}{|c|c|c|c|}
\hline Variáveis & $\begin{array}{c}\% \\
\text { resposta }\end{array}$ & $\mathbf{N}$ & $\%$ \\
\hline Grupo etário & 99,7 & & \\
\hline 60-69 anos & & 115 & 36,5 \\
\hline 70-79 anos & & 106 & 33,7 \\
\hline$\geq 80$ anos & & 94 & 29,8 \\
\hline Arranjo familiar & 100,0 & & \\
\hline Vive acompanhado & & 264 & 83,5 \\
\hline Vive sozinho & & 52 & 16,5 \\
\hline Sabe ler e escrever & 100,0 & & \\
\hline Sim & & 105 & 33,2 \\
\hline Não & & 211 & 66,8 \\
\hline $\begin{array}{l}\text { Trabalho remunerado ou } \\
\text { voluntário }\end{array}$ & 98,1 & & \\
\hline Sim & & 35 & 11,3 \\
\hline Não & & 275 & 88,7 \\
\hline Tabagismo & 99,7 & & \\
\hline Nunca fumou & & 133 & 42,2 \\
\hline Ex-fumante & & 147 & 46,7 \\
\hline Fumante & & 35 & 11,1 \\
\hline Consumo de bebidas alcoólicas & 99,7 & & \\
\hline$<1 \mathrm{dia} /$ semana & & 27 & 8,6 \\
\hline$\geq 1 \mathrm{dia} /$ semana & & 288 & 91,4 \\
\hline Sintomatologia depressiva & 91,8 & & \\
\hline Negativa & & 232 & 80,0 \\
\hline Positiva & & 58 & 20,0 \\
\hline Pressão arterial elevada & 96,8 & & \\
\hline Não & & 50 & 16,3 \\
\hline Sim & & 256 & 83,7 \\
\hline Hiperglicemia em jejum & 97,8 & & \\
\hline Não & & 273 & 88,3 \\
\hline Sim & & 36 & 11,7 \\
\hline Capacidade funcional & 97,2 & & \\
\hline Independente & & 130 & 42,3 \\
\hline Dependente em AIVD & & 126 & 41,0 \\
\hline Dependente em ABVD e AIVD & & 51 & 16,6 \\
\hline
\end{tabular}

Grande do Sul e Santa Catarina (47,3\%), e inferior à verificada em cidades da região Nordeste do País $(67,5 \%)$. Tomando como parâmetro a prevalência mundial de inatividade física $(21,4 \%)$, estimada no estudo de Dumith et al. ${ }^{28}$, a prevalência deste comportamento na população do presente estudo pode ser considerada elevada. No entanto, nos estudos citados, a idade dos indivíduos e o processo de amostragem da população foram diferentes, dificultando maiores comparações.

A associação entre a inatividade física e avanço da idade, observada no presente estudo, é consistente com os resultados de outros estudos transversais ${ }^{14,15,29,30}$. Esta associação sugere que $o$ 
Tabela 2. Prevalência de inatividade física e sua associação com as variáveis explanatórias do estudo. Lafaiete Coutinho, Brasil, 2011.

\begin{tabular}{|c|c|c|c|c|c|}
\hline Nível & Variáveis & $\%$ & RP bruta & IC95\% & $\mathbf{p}$ \\
\hline \multirow[t]{13}{*}{1} & Sexo & & & & 0,908 \\
\hline & Feminino & 46,2 & 1 & & \\
\hline & Masculino & 46,8 & 1,01 & $0,80-1,29$ & \\
\hline & Grupo etário & & & & $<0,001$ \\
\hline & 60-69 anos & 30,7 & 1 & & \\
\hline & 70-79 anos & 39,8 & 1,30 & $0,90-1,87$ & \\
\hline & $\geq 80$ anos & 72,8 & 2,37 & $1,75-3,21$ & \\
\hline & Arranjo familiar & & & & 0,505 \\
\hline & Vive acompanhado & 47,3 & 1 & & \\
\hline & Vive sozinho & 42,0 & 0,89 & $0,63-1,26$ & \\
\hline & Sabe ler e escrever & & & & 0,015 \\
\hline & Sim & 36,2 & 1 & & \\
\hline & Não & 51,7 & 1,43 & $1,07-1,90$ & \\
\hline \multirow[t]{10}{*}{2} & Trabalho remunerado ou voluntário & & & & 0,001 \\
\hline & Sim & 8,6 & 1 & & \\
\hline & Não & 51,5 & 6,00 & $2,02-17,83$ & \\
\hline & Tabagismo & & & & 0,153 \\
\hline & Nunca fumou & 40,9 & 1 & & \\
\hline & Ex-fumante & 49,0 & 1,20 & $0,92-1,56$ & \\
\hline & Fumante & 57,1 & 1,40 & $0,98-1,99$ & \\
\hline & Consumo de bebidas alcoólicas & & & & 0,059 \\
\hline & $<1 \mathrm{dia} /$ semana & 48,4 & 1 & & \\
\hline & $\geq 1 \mathrm{dia} /$ semana & 25,9 & 0,54 & $0,28-1,02$ & \\
\hline \multirow[t]{13}{*}{3} & Sintomatologia depressiva & & & & $<0,001$ \\
\hline & Negativa & 37,3 & 1 & & \\
\hline & Positiva & 61,4 & 1,65 & $1,26-2,15$ & \\
\hline & Pressão arterial elevada & & & & 0,026 \\
\hline & Não & 30,0 & 1 & & \\
\hline & Sim & 49,6 & 1,65 & $1,06-2,57$ & \\
\hline & Hiperglicemia em jejum & & & & 0,299 \\
\hline & Não & 45,6 & 1 & & \\
\hline & Sim & 54,3 & 1,19 & $0,86-1,66$ & \\
\hline & Capacidade funcional & & & & $<0,001$ \\
\hline & Independente & 29,5 & 1 & & \\
\hline & Dependente em AIVD & 54,5 & 1,85 & $1,35-2,53$ & \\
\hline & Dependente em ABVD e AIVD & 66,8 & 2,33 & $1,68-3,22$ & \\
\hline
\end{tabular}

avanço da idade é preditor da redução do nível de atividade física.

A redução nos níveis de atividade física com avanço da idade parece ser uma característica própria do processo de envelhecimento, consequente à interação entre a redução na função mitocondrial no músculo esquelético e outros fatores regulatórios ainda não conhecidos, que afetam a "motivação" dos idosos para a prática de atividade física ${ }^{31}$. Outros fatores como redução da massa muscular ${ }^{32}$ e presença de artrose e/ou artrite $^{33,34}$ dificultam ou impedem a realização de movimento, contribuindo para inatividade em idosos longevos.
A associação entre inatividade física e o envolvimento em alguma atividade ocupacional, remunerada ou não, verificada no presente estudo, pode ser explicada pela característica da ocupação. As atividades agropecuárias e o setor de serviços são as principais atividades de trabalho exercidas em Lafaiete Coutinho ${ }^{16}$. A ocupação profissional ao longo da vida de $65,4 \%$ dos entrevistados foi na agricultura/pecuária ${ }^{35}$, atividades que envolvem grande esforço físico. A influência da atividade ocupacional no nível de atividade física global deve ser interpretada com cautela, uma vez que dados de um estudo longitudinal ${ }^{36}$ sugerem que o maior nível de atividade ocupacional pode não 


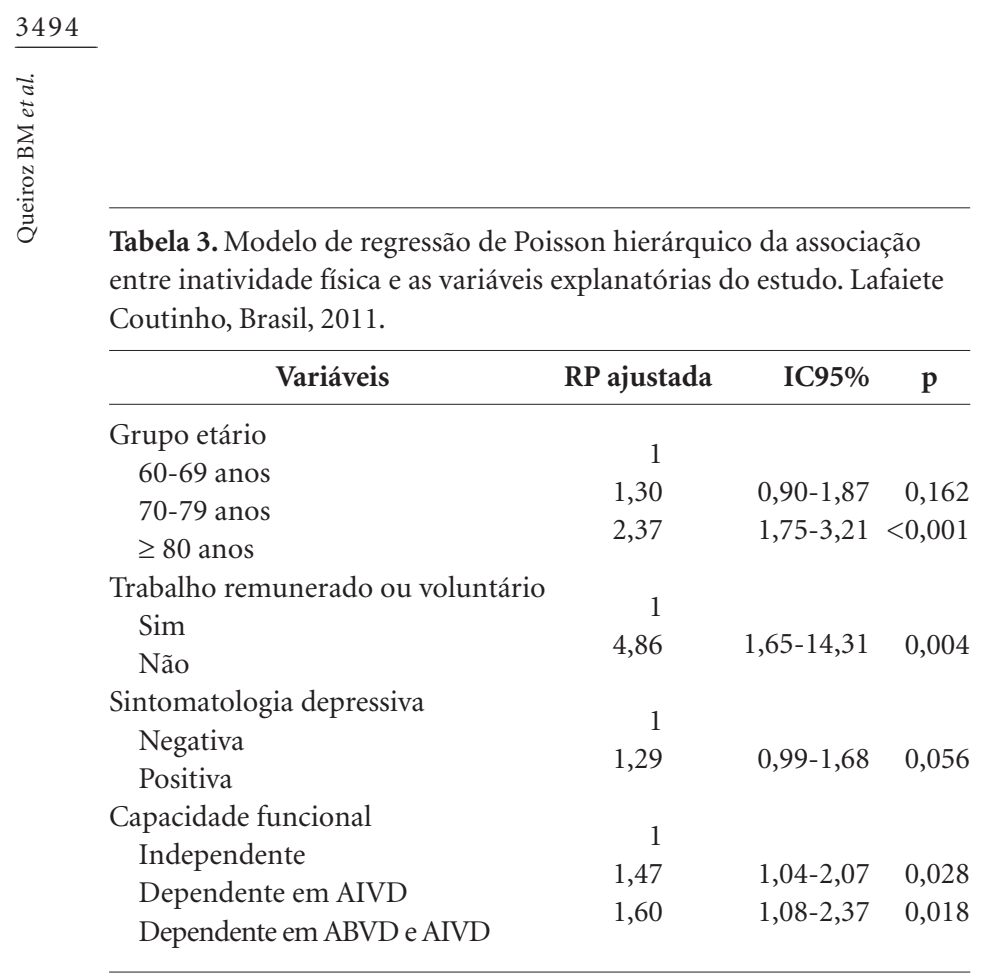

estar relacionado aos benefícios à saúde. Outros estudos devem ser incentivados a fim de que esta relação seja melhor compreendida.

Os resultados mostraram associação entre capacidade funcional e inatividade física, o que tem sido coerente com outros estudos envolvendo ido$\operatorname{sos}^{37-40}$. Virtuoso Júnior et al..$^{38}$ sugeriram pontos de cortes de atividade física para predizer a ausência de incapacidade funcional, identificando a necessidade de 280 minutos/semana para mulheres e 410

\section{Colaboradores}

RS Coqueiro, AR Barbosa e MH Fernandes foram responsáveis pela concepção e delineamento. RS Coqueiro realizou a análise e interpretação dos dados. BM Queiroz, RS Coqueiro, JS Leal Neto, AF Borgatto, AR Barbosa e MH Fernandes contribuíram na redação do artigo, revisão crítica e aprovaram a versão a ser publicada. minutos/semana para homens, valores superiores aos parâmetros de atividade física recomendados para indivíduos de 18 a 65 anos $^{39}$.

Algumas limitações do presente estudo devem ser mencionadas. O delineamento transversal dificulta o avanço em análises temporais, não permitindo identificar relação de causalidade. A utilização do instrumento para avaliação do nível de atividade física, por se tratar de uma medida indireta pode subestimar ou superestimar algumas informações. Entretanto, trata-se de instrumento simples e de fácil aplicação, amplamente utilizado em estudos populacionais ${ }^{11,15,29}$.

Em conclusão, mesmo que atividade física regular seja essencial para um envelhecimento saudável, no presente estudo a prevalência de inatividade física foi observada em quase metade da população. Os resultados deste estudo também indicaram que mais idade ( $\geq 80$ anos), ausência de ocupação e comprometimento da capacidade funcional foram preditores de inatividade física. As principais recomendações, assim como para os adultos, sugerem que indivíduos idosos se envolvam em atividades aeróbicas, de fortalecimento muscular e flexibilidade. Diante deste contexto é preciso que sejam incentivados programas específicos que estimulem a prática da atividade física, tendo em vista o combate a inatividade física e os fatores de risco decorrentes deste comportamento. Sugere-se também que outros estudos sejam realizados em populações de baixa renda, de maneira que a influência da atividade ocupacional possa ser mais bem compreendida.

\section{Agradecimentos}

À Universidade Estadual do Sudoeste da Bahia (UESB) pela bolsa de Iniciação Científica BM Queiroz recebeu. À UESB e à Fundação de Amparo à Pesquisa do Estado da Bahia - FAPESB pelo financiamento da pesquisa. À Secretaria Municipal de Saúde de Lafaiete Coutinho (BA) e aos idosos que participaram do estudo. 


\section{Referências}

1. Instituto Brasileiro de Geografia e Estatística (IBGE), Ministério do Desenvolvimento Social e Combate à Fome (MDS). Censos Demográfico de 2010: Dados preliminares do universo. Brasília: MDS; 2011 maio 3 [acessado 2012 jul 13]. Disponível em: http://www.ibge.gov.br

2. Veras R. Population aging today: demands, challenges and innovations. Rev Saude Publica 2009; 43(3):548-554.

3. Organização Mundial da Saúde (OMS), Organização Pan-Americana da Saúde (OPAS). Envelhecimento ativo: uma política de saúde. Brasília: OMS, OPAS; 2005.

4. Geib LTC. Determinantes sociais da saúde do idoso. Cien Saude Colet 2012; 17(1):123-133.

5. Ramos LR. Fatores determinantes do envelhecimento saudável em idosos residentes em centro urbano: Projeto Epidoso, São Paulo. Cad Saude Publica 2003; 19(3):793-797.

6. Warbuton DER, Nicol CW, Bredin SSD. Health benefits of physical activity: the evidence. CMAJ 2006; 174(6):801-809.

7. Löllgen H, Böckenhoff A, Knapp G. Physical activity and all-cause mortality: an updated meta-analysis with different intensity categories. Int J Sports Med 2009; 30(3):213-224.

8. Chodzko-Zajko WJ, Proctor DN, Fiatarone Singh M a Minson CT, Nigg CR, Salem GJ, Skinner JS. American College of Sports Medicine position stand. Exercise and physical activity for older adults. Med Sci Sports Exerc 2009; 41(7):1510-1530.

9. Sun F, Norman IJ, While AE. Physical activity in older people: a systematic review. BMC Public Health 2013; 13(6):449.

10. Knuth AG, Hallal PC. Temporal trends in physical activity: a systematic review. J Phys Act Health 2009; 6(5):548-559.

11. Hallal PC, Victora CG, Wells JC, Lima RC. Physical inactivity: prevalence and associated variables in Brazilian adults. Med Sci Sports Exerc 2003; 35(11):1894-1900.

12. Knuth AG, Bacchieri G, Victora CG, Hallal PC. Changes in physical activity among Brazilian adults over a 5-year period. J Epidemiol Community Health 2010; 64(7):591-595.

13. Pitanga FGD, Lessa I. Prevalência e fatores associados ao sedentarismo no lazer em adultos. Cad Saude Publica 2005; 21(3):870-877.

14. Alves JGB, Siqueira FV, Figueiroa JN, Facchini LA, Silveira DS, Piccini RX, Tomasi E, Thumé E, Hallal PC. Prevalência de adultos e idosos insuficientemente ativos moradores em áreas de unidades básicas de saúde com e sem Programa Saúde da Família em Pernambuco, Brasil Cad Saude Publica 2010; 26(3):543-556.

15. Siqueira FV, Facchini LA, Piccini RX, Tomasi E, Thumé E, Silveira DS, Hallal PC. Atividade física em adultos e idosos residentes em áreas de abrangência de unidades básicas de saúde de municípios das regiões Sul e Nordeste do Brasil. Cad Saude Publica 2008; 24(1):39-54.

16. Leal Neto JS, Coqueiro RS, Freitas RS, Fernandes MH, Oliveira DS, Barbosa AR. Anthropometric indicators of obesity as screening tools for high blood pressure in the elderly. Int J Nurs Pract 2013; 19(4):360-367.
17. Brito TA, Fernandes MH, Coqueiro RS, Jesus CS. Falls and functional capacity in the oldest old dwelling in the community. Texto \& Contexto-Enfermagem 2013; 22(1):43-51

18. Craig CL, Marshall AL, Sjostrom M Bauman AE, Booth ML, Ainsworth BE, Pratt M, Ekelund U, Yngve A, Sallis JF, Oja P. International Physical Activity Questionnaire: 12-Country Reliability and Validity. Med Sci Sports Exerc 2003; 35(8):1381-1395.

19. Katz S, Ford AB, Moskowitz RW, Jackson BA, Jaffe MW. Studies of illness in the aged. The index of ADL: a standardized measure of biological and psychosocial function. JAMA 1963; 185:914-919.

20. Almeida OP, Almeida SA. Confiabilidade da versão brasileira da escala de depressão em geriatria (GDS) versão reduzida. Arq Neuropsiquiatr 1999; 57(2B):421-426.

21. Sociedade Brasileira de Cardiologia, Sociedade Brasileira de Hipertensão, Sociedade Brasileira de nefrologia. VI Diretrizes Brasileiras de Hipertensão. Braz J Hypertens 2010; 1:1-60.

22. Coqueiro RS, Santos MC, Leal Neto JS, Queiroz BM, Brugger NAJ, Barbosa AR. Validity of a Portable Glucose, Total Cholesterol, and Triglycerides Multi-Analyzer in Adults. Biol Res Nurs 2013; 16(3):288-294.

23. Sociedade Brasileira de Diabetes. Diretrizes da Sociedade Brasileira de Diabetes 2009/Sociedade brasileira de diabetes. $3^{\text {a }}$ ed. Itapevi: A. Araújo Silva Farmacêutica; 2009.

24. Lawton MP, Brody EM. Assesment of older people: selfmaintaining and instrumental activities of daily living. Gerontologist 1969; 9(3):179-186

25. Freitas RS, Fernandes MH, Coqueiro RS, Reis Júnior WM, Rocha SV, Brito TA. Functional capacity and associated factors in the elderly: a population study. Acta Paul Enferm 2012; 25(6):933-939.

26. Hoeymans N, Feskens EJ, van den Bos GA, Kromhout D. Measuring functional status: cross-sectional and longitudinal associations between performance and self-report (Zutphen Elderly Study 1990-1993). J Clin Epidemiol 1996; 49(10):1103-1110.

27. Victora CG, Huttly SR, Fuchs SC, Olinto MTA. The role of conceptual frameworks in epidemiological analysis: a hierarchical approach. Int J Epidemiol 1997; 26(1):224-227.

28. Dumith SC, Hallal PC, Reis RS, Kohl III HW. Worldwide prevalence of physical inactivity and its association with human development index in 76 countries. Prev Med 2011; 53(1-2):41-48.

29. Boscatto EC, Duarte MFS, Barbosa AR. Nível de atividade física e variáveis associadas em idosos longevos de Antônio Carlos, SC. Rev Bras Ativ Fis e Saúde 2012; 7(2):132-136.

30. Giuli C, Papa R, Mocchegiani E, Marcellini F. Predictors of participation in physical activity for community-dwelling elderly Italians. Arch Gerontol Geriatr 2012; 54(1):50-54

31. Nair KS. Aging muscle. Am J Clin Nutr 2005; 81(5):953963 
32. Evans W. Skeletal muscle loss: cachexia, sarcopenia, and inactivity. Am J Clin Nutr 2010; 91(Supl.):1123S-1127S.

33. Artaud F, Dugravot A, Sabia S, Singh-Manoux A, Tzourio C, Elbaz A. Unhealthy behaviours and disability in older adults: Three-City Dijon cohort study. BMJ 2013; 347 (23): 4240.

34. Lêng CH, Wang JD. Long term determinants of functional decline of mobility: an 11-year follow-up of 5464 adults of late middle aged and elderly. Arch Gerontol Geriatr 2013; 57(2):215-220.

35. Fares D, Barbosa AR, Borgatto AF, Coqueiro RS, Fernandes MH. Factors associated with nutritional status of the elderly in two regions of Brazil. Rev Assoc Med Bras 2012; 58(4):434-441.

36. Holtermann A, Marott JL, Gyntelberg F, Søgaard K, Suadicani P, Mortensen OS, Prescott E, Schnohr P. Does the Benefit on Survival from Leisure Time Physical Activity Depend on Physical Activity at Work? A Prospective Cohort Study. PLoS ONE 2013: 8(1):545-548.

37. Yorston LC, Kolt GS, Rosenkranz RR. Physical activity and physical function in older adults: the 45 and up study. J Am Geriatr Soc 2012; 60(4):719-725.

38. Virtuoso Júnior JS, Tribess S, De Paulo TRS, Martins CA, Romo-Perez V.Atividade física como indicador preditivo para incapacidade funcional em pessoas idosas. Rev. Latino-Am. Enfermagem 2012; 20(2):7.

39. Haskell WL, Lee IM, Pate RR, Powell KE, Blair SN, Franklin BA, Macera CA, Heath GW, Thompson PD, Bauman A. Physical activity and public health: updated recommendation for adults from the American College of Sports Medicine and the American Heart Association. Med Sci Sports Exerc 2007; 39(8):1423-1434.

40. Virtuoso Júnior JS, Guerra RO. Incapacidade funcional em mulheres idosas de baixa renda. Cien Saude Colet 2011; 16(5):2541-2548.

Artigo apresentado em 16/10/2013

Aprovado em 27/11/2013

Versão final apresentada em 03/12/2013 